\title{
Lamprey immune protein triggers the ferroptosis pathway during zebrafish embryonic development
}

\section{Yue Pang ( $\nabla$ pangyue01@163.com )}

Liaoning Normal University

Zeyu Du

Liaoning Normal University

Duo Zhang

Liaoning Normal University

Jun Li

Liaoning Normal University

Qingwei Li

Liaoning Normal University

\section{Research Article}

Keywords: LIP, transgenic zebrafish, edema, lipid peroxidation, ferroptosis

Posted Date: February 4th, 2022

DOI: https://doi.org/10.21203/rs.3.rs-1309258/v1

License: (c) (1) This work is licensed under a Creative Commons Attribution 4.0 International License. Read Full License

Version of Record: A version of this preprint was published at Cell Communication and Signaling on August 17th, 2022. See the published version at https://doi.org/10.1186/s12964-022-00933-0. 


\section{Abstract}

Background: Previously, a novel lamprey immune protein (LIP) was identified, which plays an important role in immunity and in the regulation of growth and development in lampreys. However, the mechanism through which LIP regulates growth and development remains unclear.

Methods: Here, a zebrafish model of LIP overexpression was established through the delivery of a transgenic plasmid to the fertilized egg. The biological function of LIP was explored in vivo through phenotypic characteristics, comparative transcriptome sequencing, and physiological and biochemical analyses.

Results: LIP caused developmental toxicity in zebrafish, increasing embryo mortality and exhibiting strong teratogenic, lethal, and developmental inhibitory effects. Comparative transcriptome analysis showed that LIP-induced large-scale cell death by triggering the ferroptosis pathway. Furthermore, LIPinduced lipid peroxidation and ferroptosis trigger pericardial edema. Direct inhibition of acs/4a and tfr1a, or silencing of acs/4a and tfr1a with specific siRNA suppressed ferroptosis and pericardial edema.

Conclusions: Taken together, we confirmed that LIP can participate in growth and development via the regulation of lipid peroxidation and ferroptosis. This lays the foundation for future studies on the function of LIP in lampreys.

\section{Background}

The origin of the adaptive immune system involves profound changes from invertebrates to vertebrates (1). Lampreys belong to cyclostomes, which are among the most primitive vertebrates, and can provide unique insights into adaptive immune system origins owing to their phylogenetic position at the base of the vertebrate tree (2). In our previous studies, a novel lamprey immune protein (LIP) was identified as a highly expressed immune molecule from the supraneural body of lampreys, and the overall crystallographic structure of LIP was determined, showing an N-terminal lectin module and a C-terminal aerolysin module (3-4). The aerolysin-like protein DIn1, with similar structures, has been reported in zebrafish, suggesting a novel fish-specific defense molecule (5). As a cytotoxic protein, LIP was found to have tumor-killing activity both in vitro and in vivo. Previous studies have shown that the cytotoxic action of LIP depends on phosphatidylserine content and ROS accumulation in the cell membrane (6). In addition, LIP overexpression inhibits HeLa cell proliferation (6). To date, most studies on LIP have only focused on cytotoxic function in vitro. Recently, we demonstrated that overexpression of miR-4561 can induce apoptosis of embryonic cells by targeting LIP, suggesting a role for LIP in embryonic development (7). However, the mechanism by which LIP regulates growth and development remains unknown.

As a migratory creature, lampreys have a complex anadromous migration habit and life history, which greatly hinders the artificial breeding of lamprey. Larval lamprey live in the substrate of freshwater streams for 2-12 years before metamorphosing into their juvenile parasitic form (8). After metamorphosis, adults migrate into streams to reproduce in the spring (9), and they are highly selective 
over spawning habitats (10-11). Although lamprey species have been successfully bred and developed larvae, it is still very difficult for them to develop into adults (12-13). Hence, it is difficult to establish a suitable lamprey model for the biological function of LIP.

Among all the conventional model animals, the evolutionary status of zebrafish is closest to that of lamprey. Basic organ patterning is conserved between lamprey and zebrafish, therefore, zebrafish are used to study the functional genes and organ systems of lamprey. Compared with lampreys, zebrafish have a stable genetic background, complete genome, and simple genetic operation. Furthermore, the rich mutagenesis library accumulated by previous genetic studies on zebrafish also provides conditions for studying functional genes (14). In conclusion, the evidence suggests that the best choice for studying the function of LIP by constructing a transgenic zebrafish model.

In this study, a model of LIP-overexpressing transgenic zebrafish was established to explore the biological functions of LIP. The relationship between LIP and embryonic development is mediated by lipid peroxidation and ferroptosis. Therefore, Tg (TRE:EGFP-lip) can not only determine LIP in the physiological and pathophysiological functions of ferroptosis in vivo, but also improve our understanding of the relationship between iron metabolism and lipid metabolism in lampreys. In addition, our work can lay the foundation for studying lipid metabolism and ferroptosis by LIP in lamprey and provide new insights into lamprey growth and development.

\section{Materials And Methods}

\section{Zebrafish husbandry and drug treatments}

The AB strain of zebrafish was raised and maintained under standard laboratory conditions. Zebrafish were maintained at $28.5^{\circ} \mathrm{C}$ on a $14 \mathrm{~h}$ light $/ 10 \mathrm{~h}$ dark cycle. Embryos were maintained at $28.5^{\circ} \mathrm{C}$ in fish water $(0.2 \%$ Instant Ocean Salt in deionized water). The embryos were washed and staged according to published guidelines (15).

Zebrafish at the blastula stage were considered to have developed normally and were selected for drug treatment for eight hours post-fertilization (hpf). Dox (Doxycycline Hyclate, Yeasen Biotech, China, 30 $\mu \mathrm{g} / \mathrm{mL}$ ) was used to induce the target gene. The treated embryos were protected from light, and the culture medium was changed daily.

\section{Generation of the transgenic zebrafish line}

A total of $50 \mathrm{pg}$ pTol2-actb2-rtTAM2-TREP-EGFP-P2A-lip was microinjected into each of the one-cell stage fertilized eggs. The injected embryos were incubated at $28.5^{\circ} \mathrm{C}$ and the hatched larvae were reared to adults. Total genomic DNA from adult fish tails was extracted and amplified using two pairs of PCR primers (Table S1). The initial denaturation of DNA at $94^{\circ} \mathrm{C}$ for 5 min was followed by 35 cycles of denaturation at $94{ }^{\circ} \mathrm{C}$ for $30 \mathrm{~s}$, primer annealing at $55^{\circ} \mathrm{C}$ for $30 \mathrm{~s}$, and primer extension at $72{ }^{\circ} \mathrm{C}$ for $45 \mathrm{~s}$. 
The final elongation step was performed at $72{ }^{\circ} \mathrm{C}$ for $10 \mathrm{~min}$. PCR products were subjected to agarose gel electrophoresis.

\section{Phenotypic analysis}

The experimental samples were separated into four groups. Wild-type zebrafish embryos and transgenic zebrafish embryos were used as the control group, wild-type zebrafish embryos and transgenic zebrafish embryos at the same embryonic stage after $8 \mathrm{hpf}$ treatment with $30 \mu \mathrm{g} / \mathrm{mL}$ Dox were used as the experimental group. All zebrafish embryos were cultured in the dark at $28.5^{\circ} \mathrm{C}$ after collection. The wildtype control and experimental groups were used to determine the effect of Dox on the zebrafish phenotype, and the transgenic control and experimental groups were used to determine the effect of LIP overexpression on the zebrafish phenotype.

Before performing morphological tests, zebrafish were rinsed thoroughly in fish water three times (5 $\mathrm{min} /$ wash) and anesthetized with $0.016 \%$ MS-222 (Tricaine methanesulfonate, Sigma-Aldrich, St. Louis, $\mathrm{MO})$. Zebrafish were then oriented on their lateral side and mounted with $3 \%$ methylcellulose $\left(\mathrm{C}_{20} \mathrm{H}_{38} \mathrm{O}_{11}\right.$, Meilunbio ${ }^{\circledR}$, China) on a depression slide for observation using a stereomicroscope. The zebrafish's posture is adjusted so that it is suitable for observation, so that the eyes and body parts coincide as much as possible, and the tail and body are at the same level. The situation was observed and recorded under a stereo microscope, and the zebrafish embryo survival rate, deformity rate, and deformity were counted. In addition, the body length, eyeball radius, and yolk sac area of $4 \mathrm{dpf}$ and $7 \mathrm{dpf}$ zebrafish were recorded. The unhatched zebrafish larvae were stripped of the egg membrane before observation and recording.

The zebrafish heart is clearly visible in front of the yolk sac and behind the jaw. The heart rate of normal zebrafish is easily identified by the heartbeat. Before measuring the zebrafish larva heart rate, it was placed at room temperature $\left(28^{\circ} \mathrm{C}\right)$ to adapt to the ambient temperature for $15 \mathrm{~min}$. Zebrafish embryos were observed using a stereomicroscope and adjusted for the visibility of the heart. The number of zebrafish heartbeats was recorded every $20 \mathrm{~s}$. The number of heartbeats was repeated three times to obtain the final value of the heartbeat per minute.

\section{AO staining for cell death}

Zebrafish embryos at $24 \mathrm{hpf}, 48 \mathrm{hpf}, 72 \mathrm{hpf}$ and $96 \mathrm{hpf}$ were collected for the experiments. Zebrafish embryos were immersed in $5 \mu \mathrm{g} / \mathrm{mL}$ AO (acridinium chloride hemi-[zinc chloride], Sigma-Aldrich, St. Louis, $\mathrm{MO}$ ) in fish water for $60 \mathrm{~min}$. Next, zebrafish were rinsed thoroughly in fish water three times (5 $\mathrm{min} /$ wash) and anesthetized with $0.016 \%$ MS-222 (Tricaine methanesulfonate, Sigma-Aldrich, St. Louis, $\mathrm{MO})$. Zebrafish were then oriented on their lateral side and mounted with $3 \%$ methylcellulose $\left(\mathrm{C}_{20} \mathrm{H}_{38} \mathrm{O}_{11}\right.$, Meilunbio ${ }^{\circledR}$, China) on a depression slide for observation using a zoom stereo microscope (SMZ1500, Nikon, Japan). 
Zebrafish embryos at $19 \mathrm{hpf}, 36 \mathrm{hpf}, 60 \mathrm{hpf}$, and $96 \mathrm{hpf}$ were collected for transcriptome sequencing. RNA extraction and sequencing were performed by the Shanghai OE Biotech Co., Ltd. RNA-seq analysis was performed according to standard procedures. The DEGs between two samples were selected based on the following criteria: (1) the fold change was greater than 2 and (2) the false discovery rate (FDR) was less than 0.05 . To understand the functions of the differentially expressed genes, GO functional enrichment and KEGG pathway analysis were carried out by Goatools and KOBAS.

\section{Quantitative real-time PCR ( $q P C R$ )}

Quantitative PCR experiments were conducted using the SYBR ${ }^{\circledR}$ PrimeScript ${ }^{\text {TM }}$ RT-PCR Kit (TaKaRa, China) according to the manufacturer's protocol. Each reaction contained $1 \times$ SYBR Premix Ex Taq, $10 \mu \mathrm{M}$ of each primer, and $2 \mu \mathrm{L}$ of cDNA $(50 \mathrm{ng} / \mathrm{mL})$ in a final volume of $25 \mu \mathrm{L}$. Amplification was performed in a PCR Thermal Cycler Dice Real-Time System (TaKaRa, China) with the following parameters: initial denaturation at $95^{\circ} \mathrm{C}$ for $30 \mathrm{~s}$ to activate the DNA polymerase, followed by 40 cycles of $5 \mathrm{~s}$ at $95^{\circ} \mathrm{C}, 30 \mathrm{~s}$ at $60{ }^{\circ} \mathrm{C}$, and $30 \mathrm{~s}$ at $72{ }^{\circ} \mathrm{C}$. The gene primers and glyceraldehyde3-phosphate dehydrogenase (GAPDH)specific primers are listed in Supplement TableS1 (QPCR). GAPDH was used as an internal control. Each sample was analyzed in triplicate using the Thermal Cycler Dice Real-Time System analysis software (TaKaRa, China). The specificity of qPCR was validated using melting curve analysis.

\section{Western blot}

Proteins were extracted from cells or tissues and subjected to $8 \%$ Criterion Precast Midi Protein Gel electrophoresis. Regular western procedures were used. Antibodies used for western blotting included anti-LIP rabbit polyclonal (laboratory preparation), anti-HMGB1 rabbit polyclonal (laboratory preparation), eGFP monoclonal antibody (F56-6A1.2.3, Invitrogen), goat anti-rabbit IgG (D111018), anti-ACTB mouse monoclonal antibody (D191047), anti-TP53 rabbit polyclonal antibody (D220082), anti-ACSL3 rabbit polyclonal antibody (D261226), and anti-ACSL4 rabbit polyclonal antibody (D121771). Unlabeled antibodies were purchased from Sangon Biotech Co., Ltd. (Shanghai, China).

\section{Metabolism analysis}

There were two groups in the experiment: the control group consisted of wild-type zebrafish embryos, and the experimental group was transgenic zebrafish embryos. Tissue iron content, MDA content, GSH content, and GSSG content were measured using commercial assay kits (Beijing Solarbio Science \& Technology Co., Ltd.). Zebrafish embryo and lamprey tissue samples $(100 \mathrm{mg})$ were mechanically homogenized in $1 \mathrm{~mL}$ of the related lysis reagent for ferroptosis metabolic indicators. All the results were normalized to the corresponding total protein content.

\section{Regulation of ferroptosis in vivo}

Vitamin $E$ (Meilunbio ${ }^{\circledR}$, China) was dissolved in a minimal amount of dimethyl sulfoxide (DMSO). Vitamin $\mathrm{E}(0.1 \mathrm{mM})$ and plain DMSO were used as the treatment and negative controls, respectively. The prepared reagent was dissolved in fish water for co-culture. 
Ammonium ferric citrate (FAC, Meilunbio ${ }^{\circledR}$, China) was dissolved in water. Deferoxamine mesylate (DFO, MedChemExpress) is an iron chelator that binds free iron in a stable complex and prevents it from engaging in chemical reactions. DFO was dissolved in a minimal amount of DMSO to a concentration of $10 \mathrm{mM}$. FAC $(200 \mu \mathrm{g} / \mathrm{mL})$ and DFO $(100 \mu \mathrm{g} / \mathrm{mL}$, water-soluble) were used as positive and treatment controls, respectively. The prepared reagent was dissolved in fish water for co-culture.

\section{siRNA interference and small molecule inhibition}

SiRNAs were ordered from GenePharma (Suzhou, China), and $4 \mu \mathrm{L}$ siRNAs were microinjected into 1-cell stage zebrafish embryos. The siRNA sequences are listed in Supplement TableS1. ROSI (10 $\mu \mathrm{M}$ rosiglitazone, Meilunbio ${ }^{\circledR}$, China), a classic peroxisome proliferator-activated receptor-y agonist that has been used for ACSL4 inhibition (16). As an inhibitor of tfr, dihydroartemisinin (DHA, MedChemExpress) also induces ferroptosis, which has been used for the overexpression of LIP zebrafish (17).

\section{Image acquisition and statistical analysis}

Embryos and larvae were analyzed using a fluorescence microscope (SMZ1500, Nikon, Japan) and subsequently photographed using digital cameras. Quantitative image analyses were performed using image-based morphometric analysis (NIS-Elements D3.1, Nikon, Japan) and ImageJ software (National Institutes of Health, Bethesda, MD, USA). Statistical analysis and graphical representation of the data were performed using GraphPad Prism 8.0 (GraphPad Software, San Diego, CA, USA).

\section{Results}

\section{Identification and character of a LIP transgenic zebrafish model}

To create a stable LIP transgenic zebrafish line with ubiquitous LIP expression, transgenic zebrafish were established based on the TetOn system for drug-induced overexpression of LIP (Figure 1A). The Tol2actb2-rtTAM2-TREP-EGFP-P2A-lip was microinjected into one-cell stage fertilized embryos. The transgenic zebrafish were treated with Dox after $4 \mathrm{dpf}$, and high expression of green fluorescent protein appeared in the LIP transgenic zebrafish line (Figure 1B). These larvae were reared into adulthood, and the genomic DNA from their tail fins was isolated for positive screening of the lip gene. The positive rate of the lip gene in F0 fish was $6.33 \%$ (Table S2). These positive F0 fish were individually mated with wildtype fish to obtain their F1 offspring. F2 offspring with more stable fluorescence expression were obtained by self-crossing positive F1 fish. As shown in Figure 1C, PCR detection indicated that the lip gene was integrated into a single chromosomal locus. Expression of LIP and GFP was detected by western blot assay, and transgenic zebrafish had both high levels of GFP and LIP expression (Figure 1D), indicating that the lip gene is stably inherited in the progeny of zebrafish. Fluorescence detection of different generations showed that the F4 generation obtained a stable line (Figure 1E, Table S2).

\section{Overexpression of lip gene inhibits embryonic development of zebrafish}


For 4-7dpf wild-type and transgenic zebrafish, the survival rate of LIP-overexpressing zebrafish with Dox treatment was lower in the experimental group than in the LIP-overexpressing zebrafish without Dox treatment (Figure 2A). In contrast, the malformation rate of LIP-overexpressing zebrafish with Dox treatment was higher (Figure 2B), indicating that LIP overexpression is lethal or malformation in zebrafish. In addition, no abnormal morphology or pericardial edema was observed in the wild-type (Figure 2C), and severe morphological and developmental abnormalities were observed in transgenic zebrafish treated with Dox, such as spinal curvature, yolk sac edema, pericardium edema, and unhatched larvae (Figure 2C, D). In the phenotypic analysis, edema was more common than in other malformations (Figure 2D). In addition, HE staining revealed that elevated pathology was also evident in LIPoverexpressing larvae in which edema and spinal curvature were increased (Figure 2E). Furthermore, the growth and development of zebrafish showed that LIP overexpression caused significant growth retardation, including body length (Figure $2 \mathrm{~F}, \mathrm{G}$ ), eyeball radius (Figure $2 \mathrm{H}$ ), and yolk sac area (Figure 2I) compared with transgenic zebrafish without Dox treatment and the wild-type. LIP inhibited the development of body length and eyeball radius of $4 \mathrm{dpf}$ zebrafish more than $7 \mathrm{dpf}$ zebrafish, but transgenic zebrafish at 7dpf had a greater effect on yolk sac absorption than at $4 \mathrm{dpf}$. Collectively, LIP inhibits zebrafish development, including developmental retardation, teratogenicity, and embryonic lethality.

\section{LIP Overexpression causes massive cell death in the heart}

To determine the relationship of LIP with pericardial edema, heart rate was recorded in the whole process of Dox treatment. Our results demonstrated that heart rate fluctuations were not significantly different from 48 to $120 \mathrm{hpf}$ in wild-type zebrafish, but heart rate fluctuations in LIP-overexpressing transgenic zebrafish showed a significant increment at 48 hpf, 72 hpf, 96 hpf (Figure 3A). This indicates that LIP overexpression damages the heart function of zebrafish. Then, cell death was detected by acridine orange (AO) in transgenic zebrafish and wild-type zebrafish with Dox or without Dox treatment (Figure $3 \mathrm{~B}$ ), and cell death particle numbers in transgenic zebrafish with Dox treatment significantly increased in whole embryos compared to transgenic zebrafish without Dox treatment (Figure 3C). In addition, the number of apoptotic particles in the hearts of transgenic zebrafish with Dox treatment was obviously upregulated, especially at $48 \mathrm{hpf}$, and the heart was fully developed (Figure 3D). These data showed that LIP plays an inhibitory role in the growth and development of zebrafish via the control of heart function.

\section{Effect of LIP overexpression on zebrafish heart function by Ferroptosis pathway}

To further explore the role of LIP in embryonic development, we used transcriptome analysis, combined with functional validation, to reveal the potential biological functions of LIP from the perspective of functional genomics. Based on the embryonic development process, RNA-seq was performed with transgenic zebrafish at four relatively discrete stages, including myocardial progenitor cells forming a horseshoe-shaped structure (19 hpf, segmentation period), the heart begins to loop (36 hpf, hatching period), heart fully formed (60 hpf, hatching period), and complete most of its morphogenesis ( $96 \mathrm{hpf}$, early larvae). Transcriptome sequencing data are standardized data after verification by fragments per 
kilobase per million (FPKM) (Figure 4A). Following the screening standard, 1129, 614, 819, and 637 DEGs were obtained in comparisons between the wild-type and transgenic groups at different developmental stages, respectively. Among these DEGs, there were 889 upregulated and 240 downregulated genes, 288 upregulated and 327 downregulated genes, 527 upregulated and 292 downregulated genes, and 340 upregulated and 297 downregulated genes, respectively (Figure 4B). A total of 109 of the same DEGs were obtained in all four groups of wild-type and transgenic zebrafish (Figure S1A). To reveal the dynamic change of LIP across different stages, we performed PCA (Figure S1B) and showed a continuous developmental process from lip gene expression in embryos (19 hpf) to the end of embryonic development (96 hpf). Differential gene expression analysis showed that different groups were enriched in functions that corresponded to features of LIP at different stages (Figure S1C).

Comparing the commonality of the four sets of transcriptomes, we found that LIP not only disturbs lipid metabolism through the PPAR signaling pathway, but also causes cell death, such as the ferroptosis and autophagy pathways. Subsequent Gene Ontology (GO) terms and Kyoto Encyclopedia of Genes and Genomes (KEGG) pathway enrichment analyses suggested a potential impact of LIP protein overexpression on metabolic pathways involving lipid metabolism and cell death (Figure S1, S2). The ferroptosis pathway showed the strongest enrichment, followed by cogent signals that supported the KEGG analysis, including upregulation of ferroptosis marker molecules tf, tfr1a, and acsl4a. We then designed and analyzed the network of molecular interactions of ten major pathways differentially expressed in LIP transgenic zebrafish, which were divided into three groups: mitophagy, lysosome, and ferroptosis. We found that these 10 pathways were related to the ferroptosis pathway (Figure 4C). Damage to mitochondria and lysosomes is a manifestation of ferroptosis, which is a form of cell death caused by lipid metabolism. Furthermore, molecules related to ferroptosis were significantly upregulated in the overexpressed LIP transcriptome (Figure 4D). Therefore, we speculated that LIP triggered ferroptosis during embryonic development in zebrafish. The upregulation of ferroptosis-specific genes was confirmed by qPCR (Figure 4E), which validated the reliability of our transcriptome-wide data.

\section{LIP overexpression in zebrafish larvae causes edema by triggering ferroptosis}

Here, we confirmed that LIP overexpression improved the expression of both ACSL4, TP53, and HMGB1 at the protein level by immunoblotting (Figure 5A). In addition, the contents of iron (Figure 5B) and MDA (Figure 5C) in overexpressed LIP zebrafish were higher than those in the control group, while the ratios of GSH and GSH/GSSG (Figure 5D) were lower than those in the control group. The results show that LIP triggers ferroptosis by modulating GSH metabolism, iron metabolism, and lipid peroxidation at multiple levels.

We then quantified the zebrafish phenotype, ranging from P1 = normal phenotype to $\mathrm{P} 4$ = very severe edema. More than $60 \%$ of LIP-overexpressing zebrafish developed a severe ( $\mathrm{P} 3=40.33 \%$ ) or very severe edema phenotype ( $\mathrm{P} 4=23 \%$, Figure $5 \mathrm{E}$ ). To investigate whether the edema phenotype was caused by ferroptosis, we used vitamin $\mathrm{E}$ as an inhibitor of ferroptosis in transgenic Tg (TRE:EGFP-lip) zebrafish that treat the edema phenotype. In vitamin E-treated zebrafish, a significant increase in survival and edema 
phenotypes were significantly lower than that in the untreated group, indicating that LIP overexpression in zebrafish larvae causes edema by triggering ferroptosis (Figure $5 F, G$ ). To rule out that the observed phenotype was due to VE interacting with LIP, ferric ammonium citrate (FAC) and desferrioxamine (DFO) were used to regulate ferroptosis based on iron levels (Figure $5 \mathrm{H}$ ). FAC, as a ferroptosis-inducing factor, induces cell death, whereas DFO, as an iron-chelating agent, reduces the iron content in the body. Our results demonstrated that FAC caused edema in zebrafish, while DFO significantly inhibited the edema phenotype caused by FAC and LIP overexpression. Furthermore, we examined cardiomyocyte ultrastructure in vivo and found extensive mitochondrial edema with obvious ferroptosis features (Figure 5I). Therefore, LIP overexpression in zebrafish larvae causes edema by triggering ferroptosis.

\section{Intervention of tfr1a and acs/4a to inhibit ferroptosis induced by LIP overexpression}

TFR1 and ACSL4 are markers of ferroptosis and are upregulated in ferroptosis induced by LIP overexpression (18-19). To identify the direct target of LIP, we designed three types of siRNAs for tfr1a and acs $14 \mathrm{a}$. Through qPCR detection of $24 \mathrm{hpf}$ zebrafish embryos, we determined that siRNA tfr1a-799 and siRNA acs|4a-275 worked best (Figure S3C-F), therefore, they were selected for follow-up experiments.

As shown in Figure 6A-E, knocking down two key ferroptosis genes, tfr1a and acs 44 a, inhibited the edema phenotype of zebrafish larvae caused by ferroptosis caused by LIP overexpression to a certain extent. Among them, siRNA acsI4a had a significant inhibitory effect (Figure 6F-H), which is characterized by high embryo survival rate, a significant decrease in severe edema phenotype, and a significant decrease in lip transcription level. It should be noted that the mixed injection of the two siRNAs had a more significant inhibitory effect on the edema phenotype. This suggests that ferroptosis caused by LIP overexpression can be inhibited by targeting tfr1a or acs $14 a$, and acs 44 a may be the direct target of LIP. In addition, the mixed injection of two siRNAs also obtained better results, indicating that LIP acts directly on acsl4a and indirectly on tfr1a (Figure 6I-L).

To determine the regulatory effect of ACSL4 on ferroptosis triggered by LIP, ROSI was selected as a smallmolecule inhibitor of acs/4a (20). Compared with the control group, the expression level of the acs $/ 4 a$ gene was inhibited in the ROSI group (Figure 7B). The results of qPCR showed that the lip was also significantly downregulated and positively correlated with acs/4a. At the same time, inhibition of acs/4a resulted in the downregulation of lip, indicating that LIP acts directly on acs/4a (Figure 7C). Meanwhile, the abnormal edema phenotype of zebrafish embryos caused by LIP overexpression decreased significantly. The results showed that inhibiting the expression of acs/4a in zebrafish embryos had a protective effect on ferroptosis caused by overexpression of LIP, and could reduce the edema phenotype caused by overexpression of LIP (Figure 7D, E). Using dihydroartemisinin (DHA) as an inducer of ferroptosis, similar experimental results were obtained (Figure S4).

\section{Discussion}


Our previous studies focused on the cytocidal activity of LIP against tumors. Additionally, LIP is involved in embryonic development through miR-4561 regulation, however, the mechanism by which LIP regulates development remains unclear. Thus, the construction of the LIP transgenic zebrafish model was used to explore the mechanism of LIP regulation on growth and development. Phenotypic and transcriptomic analyses of transgenic zebrafish Tg(TRE:EGFP-lip), LIP inhibits growth and development rather than participates only in immune defense. Here, we demonstrate a novel mechanism to regulate development by triggering ferroptosis in LIP. In particular, the regulation of ferroptosis by LIP suggests that it has a regulatory function in iron and lipid metabolism.

LIP inhibition developments, such as embryonic lethality, abnormal morphogenesis of multiple tissues, and severe growth retardation, are critical to the survival of zebrafish embryos. For example, a reasonable explanation for edema of LIP may be that trigger ferroptosis resulted in accumulation of tissue fluid or prostaglandins, eventually leading to edema (21-22). The resting heart rate is the most sensitive indicator of cardiac function (23). LIP overexpression can greatly increase heart rate and cause cell death in the heart of zebrafish. The faster the heartbeat, the shorter the interval, the more susceptible the heart is to damage (24). The occurrence of most congenital heart diseases is closely related to embryonic cell disorders (25). Excessive ROS caused by oxidative stress are mostly produced in tissue cells with important functions, high activity, and high oxygen consumption, such as cardiac muscle cells, brain cells, and nerve cells, which makes the local free radical level of these cells much higher than that of other tissue cells, which in turn increases the occurrence of cell death (26). It is speculated that LIP overexpression causes ROS explosion and oxidative damage, which further leads to cell death and pericardial edema.

The first evidence that LIP is involved in iron metabolism came from transcriptome studies of LIP overexpression in transgenic zebrafish. Pathways associated with the cardiovascular system, such as cardiac muscle contraction pathways, are downregulated. Simultaneously, pathways related to lipid metabolism, such as the PPAR pathway, are also regulated (27). Glucose and lipid metabolism disorders are the biggest threats to cardiovascular diseases (28). This may be related to the inhibition of LIP on yolk granules and pericardial edema. Furthermore, LIP appears to influence the functions of other pathways through the ferroptosis pathway (29). Ferroptosis is a new type of iron-dependent programmed cell death that is different from apoptosis, cell necrosis, and autophagy (30). The main mechanism of ferroptosis is that under the action of divalent iron or ester oxygenase, the unsaturated fatty acids with high expression on the cell membrane are catalyzed to produce lipid peroxidation, thus inducing cell death (31). In addition, it is also manifested in the reduction of the core enzyme GPX4 in the regulation of the antioxidant system (glutathione system) (32). Previous studies have shown that ACSL4 leads to an outbreak of lipid peroxidation through the PPAR pathway (33). TFR1 promotes iron overload via the ferroptosis pathway (34). Our study demonstrated that LIP is involved in the regulation of acs 14 a and tfr1a, and thus regulates ferroptosis. P53 also regulates ferroptosis through transcriptional or posttranslational mechanisms (35). This may be related to the tumor-killing activity of the LIP. Thus, it is speculated that LIP plays an important role in immune defense and inhibits growth and development by regulating lipid metabolism and iron metabolism. MDA is a marker of lipid peroxidation, and the 
occurrence of lipid peroxidation can be determined by detecting MDA (36-37). Our results showed that high levels of iron and lipid peroxidation were detected in LIP-overexpressing transgenic zebrafish through the detection of MDA.

The inherent characteristics of ferroptosis suggest that it regulates tissue homeostasis and growth. Studies have demonstrated that ferroptosis is triggered by degenerative processes or may be induced therapeutically in some cancers, but few studies have explored its natural functions (38). Compared with other fish, lampreys feed on iron-rich blood (39). Consequently, lampreys are more prone to age-related iron accumulation (40). To counter this risk, LIP may trigger ferroptosis to maintain the iron balance in the body. It is well known that the liver is a major iron reservoir, and the liver of lampreys is rich in iron and fat, suggesting that lampreys require special mechanisms to inhibit hepatofibrosis and fatty liver (41). Zhang et al. found that ferroptosis in HSCs can effectively inhibit hepatofibrosis (42). Ferroptosis involves lipid and iron metabolism, which are closely related to growth and development. Overexpression of LIP mobilizes a large amount of stored iron, resulting in increased blood iron concentration, growth inhibition, and triggering ferroptosis. The consumption of iron may cause iron deficiency (ID), which causes nutritional anemia and may also contribute to poor development (43). In addition, ferroptosis leads to myocarditis, which is associated with the pericardial edema phenotype in zebrafish (44). Simultaneously, iron imbalance can lead to abnormal bone metabolism, which is related to the spine curvature phenotype of zebrafish (45). Clarification of the definitive role of LIP in ferroptosis will be very helpful to our understanding of the origin and function of LIP. Although research has revealed that LIP is related to immune recognition and defense, the role of LIP in the growth and development of lampreys remains unclear. It is of great significance to clarify the specific role of LIP in triggering ferroptosis, as it helps to explain the dual role of LIP in immune defense and growth.

\section{Conclusions}

In summary, our data constructed a transcriptome of LIP transgenic zebrafish and demonstrated that LIP inhibits embryo development by triggering the ferroptosis pathway, providing an animal model for studying LIP and ferroptosis. Importantly, we elucidated that LIP directly targets acs/4 and mediates lipid peroxidation to trigger ferroptosis. Considerable progress has been made regarding the biological functions of LIP in lampreys. A schematic illustration of LIP functions is shown in Figure 8. Further elucidation of the exact molecular mechanisms of ferroptosis and developmental delay caused by LIP will help us understand the basic processes of iron metabolism, which may contribute to the development of new treatments for hemochromatosis and developmental and neurodegenerative diseases.

\section{Abbreviations}

LIP: lamprey immune protein, ROS. reactive oxygen species, miR-4561: MicroRNA-4561, Dox: Doxycycline Hyclate, hpf/dpf: hour/day post-fertilization, MS-222: Tricaine methanesulfonate, AO: acridinium chloride hemi-[zinc chloride], FDR : false discovery rate, GAPDH: glyceraldehyde-3-phosphate dehydrogenase, HMGB1: high mobility group box-1 protein, eGFP: enhanced green fluorescent protein, ACTB: $\beta$-Actin, 
TP53: Tumor Protein P53, ACSL3: Acyl-CoA Synthetase Long Chain Family Member 3, ACSL4: Acyl-CoA Synthetase Long Chain Family Member 4, MDA: Malondialdehyde, GSH: glutathione, GSSG: L-Glutathione Oxidized, DMSO: dimethyl sulfoxide, FAC: Ammonium ferric citrate, DFO: Deferoxamine mesylate, ROSI: rosiglitazone, DHA: dihydroartemisinin, FPKM: fragments per kilobase per million, DEGs: differentially expressed genes, TFR1: transferrin receptor 1,GPX4: glutathione peroxidase 4 .

\section{Declarations}

\section{Authors' contributions}

Yue Pang and Zeyu Du designed research, Zeyu Du, and Duo Zhang performed research, Jun Li contributed zebrafish material/microinjection methods, Yue Pang, Zeyu Du, and Qing Wei Li analyzed data and prepared figures, and Yue Pang, and Zeyu Du wrote the paper.

\section{Funding}

Supported by the National Natural Science Foundation of China(Nos.31772884 32070518), Liaoning Climbing Scholar, the Distinguished Professor of Liaoning (No.XLYC2002093), the Program of Science and Technology of Liaoning Province (No.2019-MS-218), the Project of the Educational Department of Liaoning Province(No.LJ2020012), and the Science and Technology Innovation Fund Research Project of Dalian City (No. 2018J12SN079).

\section{Availability of data and materials}

All data generated or analysed during this study are included in this published article [and its Additional files].

\section{Ethics approval and consent to participate}

The animal experiments were performed in accordance with the regulations of the Animal Welfare and Research Ethics Committee of the Institute of Dalian Medical University's Animal Care protocol (Permit Number: SCXK2008-0002).

\section{Data availability statement}

All relevant data are available from the corresponding author upon reasonable request. The datasets presented in this study can be found in online repositories. The names of the repository/repositories and accession number(s) can be found below: NCBI, PRJNA782739.

\section{Competing interests}

The authors declare that they have no competing interests.

\section{Author details}


${ }^{1}$ College of Life Science, Liaoning Normal University, Dalian 116081, China, ${ }^{2}$ Lamprey Research Center, Liaoning Normal University, Dalian 116081, China, ${ }^{3}$ Collaborative Innovation Center of Seafood Deep Processing, Dalian Polytechnic University, 116023, China.

\section{References}

1. Cooper MD, Alder MN. The evolution of adaptive immune systems (2006). Cell. 2006, Feb 24,124(4):815-22.

2. Parker, H. J., Bronner, M. E., \& Krumlauf, R. (2014). A Hox regulatory network of hindbrain segmentation is conserved to the base of vertebrates. Nature. 2014, 514(7523), 490-493.

3. Pang Y, Gou M, Yang K, et al. Crystal structure of a cytocidal protein from lamprey and its mechanism of action in the selective killing of cancer cells. Cell Commun Signal. 2019, May 27,17(1):54.

4. Pang $\mathrm{Y}, \mathrm{Li} \mathrm{C}$, Wang $\mathrm{S}$, et al. A novel protein derived from lamprey supraneural body tissue with efficient cytocidal actions against tumor cells. Cell Commun Signal. 2017, Oct 16,15(1):42.

5. Jia N, Liu N, Cheng W, et al. Structural basis for receptor recognition and pore formation of a zebrafish aerolysin-like protein. EMBO Rep. 2016, 17:235-48.

6. Chi X, Su P, Bi D, et al. Lamprey immune protein-1 (LIP-1) from Lampetra japonica induces cell cycle arrest and cell death in HeLa cells. Fish Shellfish Immunol. 2018, Apr, 75:295-300.

7. Ma L, Gou M, Du Z, et al. MicroRNA expression profile in Lampetra morii upon Vibrio anguillarum infection and miR-4561 characterization targeting lip. Commun Biol. 2021, Aug 20,4(1):995.

8. Bussy U, Chung-Davidson YW, Buchinger T, et al. Metabolism of a sea lamprey pesticide by fish liver enzymes part B: method development and application in quantification of TFM metabolites formed in vivo. Anal Bioanal Chem. 2018, Feb,410(6):1763-1774.

9. Manion PJ, Hanson LH. Spawning behavior and fecundity of lampreys from the upper three Great Lakes. Can J Fish Aquat Sci. 1980, 37:1635-1640.

10. Teeter J. Pheromone communication in sea lampreys (Petromyzon marinus): Implications for population management. Can J Fish Aquat Sci.1980, 37: 2123- 2132.

11. Moore HH, Schleen IP. Changes in spawning runs of sea lamprey (Petromyzon marinus) in selected streams of lake superior after chemical control. Can J Fish Aquat Sci.1980, 37:1851-1860.

12. Richardson MK, Admiraal J, Wright GM. Developmental anatomy of lampreys. Biol Rev Camb Philos Soc.2010, 85: 1-33.

13. Campo-Paysaa F, Jandzik D, Takio-Ogawa Y, et al. Evolution of retinoic acid receptors in chordates: insights from three lamprey species, Lampetra fluviatilis, Petromyzon marinus, and Lethenteron japonicum. EvoDevo. 2015, 6:18.

14. Blackburn PR, Campbell JM, Clark KJ, et al. The CRISPR system-keeping zebrafish gene targeting fresh. Zebrafish. 2013, Mar,10(1):116-8. 
15. Kimmel CB, Ballard WW, Kimmel SR, et al. Stages of embryonic development of the zebrafsh. Dev Dyn. 1995, 203:253-310.

16. Li Y, Feng D, Wang Z, et al. Ischemia-induced ACSL4 activation contributes to ferroptosis-mediated tissue injury in intestinal ischemia/reperfusion. Cell Death Differ. 2019, Nov,26(11):2284-2299.

17. Lin R, Zhang Z, Chen L, et al. Dihydroartemisinin (DHA) induces ferroptosis and causes cell cycle arrest in head and neck carcinoma cells. Cancer Lett. 2016, Oct 10,381(1):165-75.

18. Song $X$, Xie $Y$, Kang R, et al. FANCD2 protects against bone marrow injury from ferroptosis. Biochem Biophys Res Commun. 2016, Nov 18,480(3):443-449.

19. Doll S, Proneth B, Tyurina YY, et al. ACSL4 dictates ferroptosis sensitivity by shaping cellular lipid composition. Nat Chem Biol. 2017, Jan,13(1):91-98.

20. Wei S, Qiu T, Wang N, et al. Ferroptosis mediated by the interaction between Mfn2 and IREa promotes arsenic-induced nonalcoholic steatohepatitis. Environ Res. 2020, Sep, 188: 109824.

21. Kong Z, Liu R, Cheng Y. Artesunate alleviates liver fibrosis by regulating ferroptosis signaling pathway. Biomed Pharmacother. 2019, Jan,109:2043-2053.

22. Wang $T$, Zhang $X$, Ye Y, et al.. Quantification of prostaglandins $E 2$ and D2 using liquid chromatography-tandem mass spectrometry in a mouse ear edema model. Anal Methods. 2020 Jun 11,12(22):2865-2871.

23. Böhm M, Reil JC, Deedwania P, et al.. Resting heart rate: risk indicator and emerging risk factor in cardiovascular disease. Am J Med. 2015, Mar,128(3):219-28.

24. Yalcin HC, Amindari A, Butcher JT, et al. Heart function and hemodynamic analysis for zebrafish embryos. Dev Dyn. 2017, Nov,246(11):868-880.

25. Hallaq $\mathrm{H}$, Pinter $\mathrm{E}$, Enciso J, et al. A null mutation of Hhex results in abnormal cardiac development,defective vasculogenesis and elevated Vegfa levels. Development. 2004, 131 (20):5197-5209.

26. Das DK, Maulik N. Preconditioning potentiates redox signaling and converts death signal into survival signal.Arch Biochem Biophys. 2003, 420:305-311.

27. Hirschhorn T, Stockwell BR. The development of the concept of ferroptosis. Free Radic Biol Med. 2019, Mar, 133:130-143.

28. Alizargar J, Bai CH, Hsieh NC, et al. Use of the triglyceride-glucose index (TyG) in cardiovascular disease patients. Cardiovasc Diabetol. 2020, Jan 15,19(1):8.

29. Stockwell BR, Friedmann Angeli JP, Bayir H, Bush Al, Conrad M, Dixon SJ, Fulda S, Gascón S, Hatzios SK, Kagan VE, et al. Ferroptosis: A Regulated Cell Death Nexus Linking Metabolism, Redox Biology, and Disease. Cell. 2017, Oct 5,171(2):273-285.

30. Ashrafizadeh M, Mohammadinejad R, Tavakol S, et al. Autophagy, anoikis, ferroptosis, necroptosis, and endoplasmic reticulum stress: Potential applications in melanoma therapy. J Cell Physiol. 2019, Nov, 234(11): 19471-19479. 
31. Yang WS, Kim KJ, Gaschler MM , et al. Peroxidation of polyunsaturated fatty acids by lipoxygenases drives ferroptosis. Proc Natl Acad Sci U S A. 2016, Aug 23, 113(34): E4966-75.

32. Dixon SJ, Lemberg KM, Lamprecht MR, et al. Ferroptosis: an iron-dependent form of nonapoptotic cell death. Cell. 2012, May 25,149(5):1060-72.

33. Fan Y, Han Z, Lu X, et al. Identification of Milk Fat Metabolism-Related Pathways of the Bovine Mammary Gland during Mid and Late Lactation and Functional Verification of the ACSL4 Gene. Genes (Basel). 2020, Nov 16,11(11):1357.

34. Ashok A, Chaudhary S, McDonald D, et al.Local synthesis of hepcidin in the anterior segment of the eye: A novel observation with physiological and pathological implications. Exp Eye Res. 2020, Jan,190:107890.

35. Kang R, Kroemer G, Tang D. The tumor suppressor protein p53 and the ferroptosis network. Free Radic Biol Med. 2019, Mar,133:162-168.

36. Magtanong L, Dixon SJ. Ferroptosis and Brain Injury. Dev Neurosci. 2018, 40(5-6):382-395.

37. Umeno A, Biju V, Yoshida Y. In vivo ROS production and use of oxidative stress-derived biomarkers to detect the onset of diseases such as Alzheimer's disease, Parkinson's disease, and diabetes. Free Radic Res. 2017, Apr,51(4):413-427.

38. Qiu Y, Cao Y, Cao W, et al. The Application of Ferroptosis in Diseases. Pharmacol Res. 2017, Sep,159:104919.

39. Sunga J, Wilson JM, Wilkie MP. Functional re-organization of the gills of metamorphosing sea lamprey (Petromyzon marinus): preparation for a blood diet and the freshwater to seawater transition. J Comp Physiol B. 2020, Nov,190(6):701-715.

40. Youson JH, Sargent PA. Iron deposition in the integument of lampreys. Anat Rec. 1984, Aug,209(4):461-8.

41. Macías-Rodríguez RU, Inzaugarat ME, Ruiz-Margáin A, et al. Reclassifying Hepatic Cell Death during Liver Damage: Ferroptosis-A Novel Form of Non-Apoptotic Cell Death? Int J Mol Sci. 2020, Feb 28,21(5):1651.

42. Zhang $Z$, Yao $Z$, Wang $L$, et al. Activation of ferritinophagy is required for the RNA-binding protein ELAVL1/HuR to regulate ferroptosis in hepatic stellate cells. Autophagy. 2018, 14(12):2083-2103.

43. Latunde-Dada GO. Ferroptosis: Role of lipid peroxidation, iron and ferritinophagy. Biochim Biophys Acta Gen Subj. 2017, Aug,1861(8):1893-1900.

44. Jacobs W, Lammens $M$, Kerckhofs $A$, et al. Fatal lymphocytic cardiac damage in coronavirus disease 2019 (COVID-19): autopsy reveals a ferroptosis signature. ESC Heart Fail. 2020, Sep 22,7(6):377281.

45. Xu W, Yu R, Zhu X, et al. Iron-Chelating Agent Can Maintain Bone Homeostasis Disrupted by Iron Overload by Upregulating Wnt/Beta-Catenin Signaling. Biomed Res Int. 2020, May 24,2020:8256261

\section{Figures}


A
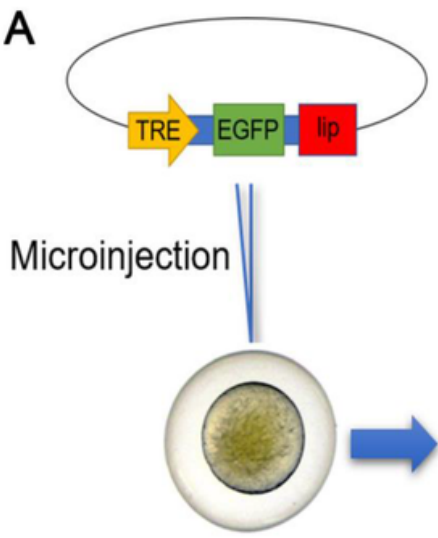

F0 embryos
B

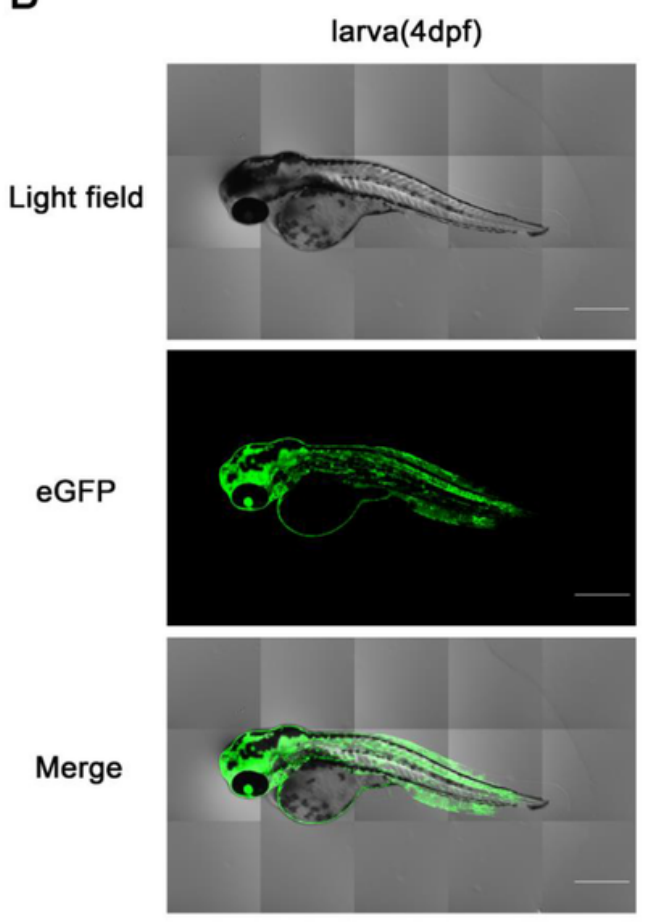

Positive F1

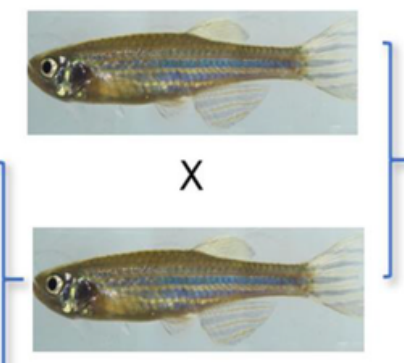

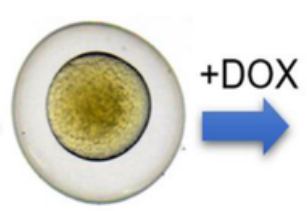

F2 embryos

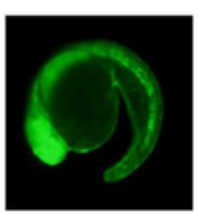

24hpf

Positive F1

\section{Positive F0}

C

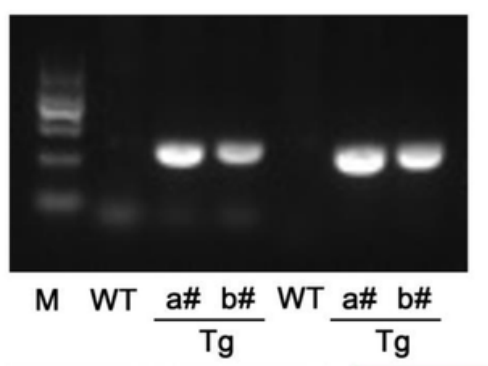

E

F2

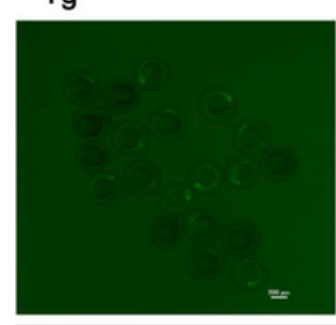

D eGFP

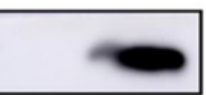

$27 \mathrm{kDa}$

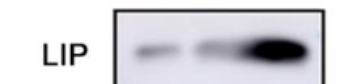

$34 \mathrm{kDa}$

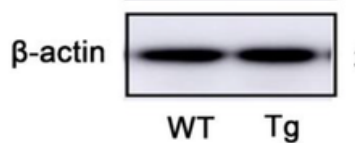
$36 \mathrm{kDa}$

F3

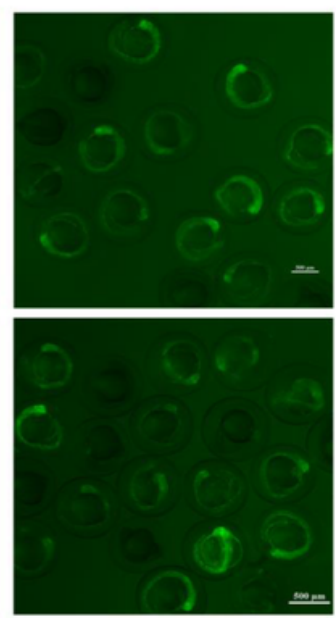

\section{Figure 1}

Establishment of a LIP-overexpression transgenic zebrafish model. (A) The plasmid was microinjected into fertilized wild-type (WT) eggs at one-cell stage. The positive F0 fish was individually crossed with WT fish to obtain positive F1 fish. F2 fish was reproduced by selfing the positive F1. Imaging of EGFP expression in F2 embryos with or without Dox was performed by using a fluorescence microscope. (B) Confocal imaging overexpression of fluorescent expression of LIP zebrafish. Scale 50 $\mu$ m. (C) Positive F2 individuals were obtained by PCR screening. M: DNA ladder, lanes a\# and b\#: individual DNA samples (two transgenic lines with fluorescent expression obtained). The positive sample gave rise to a 367-374

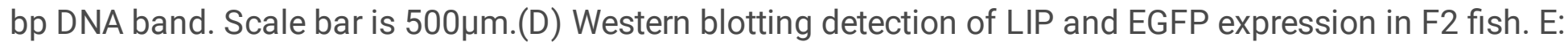
Fluorescent expression efficiency of F2 and F3 generation transgenic zebrafish. 

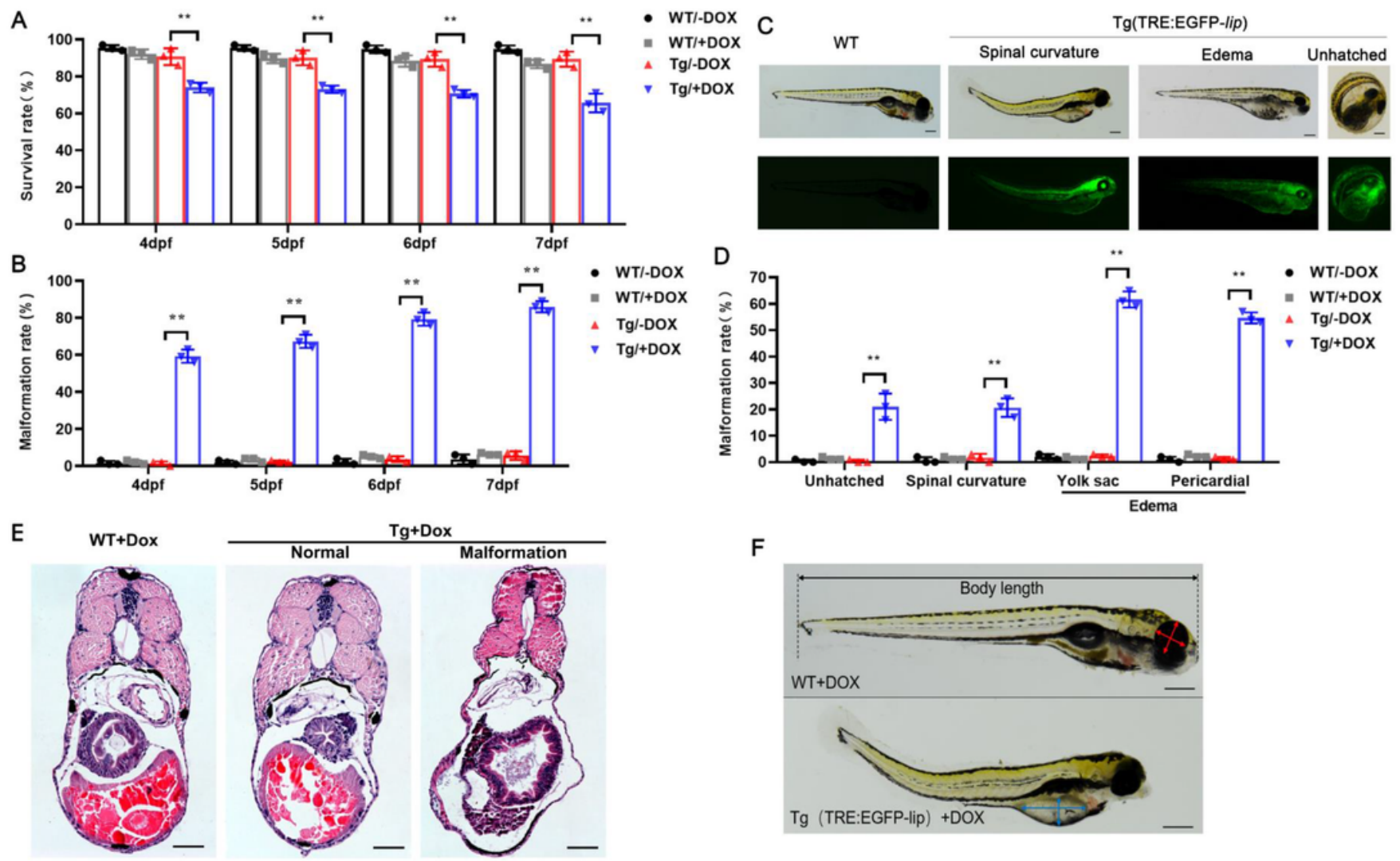

$\mathrm{F}$
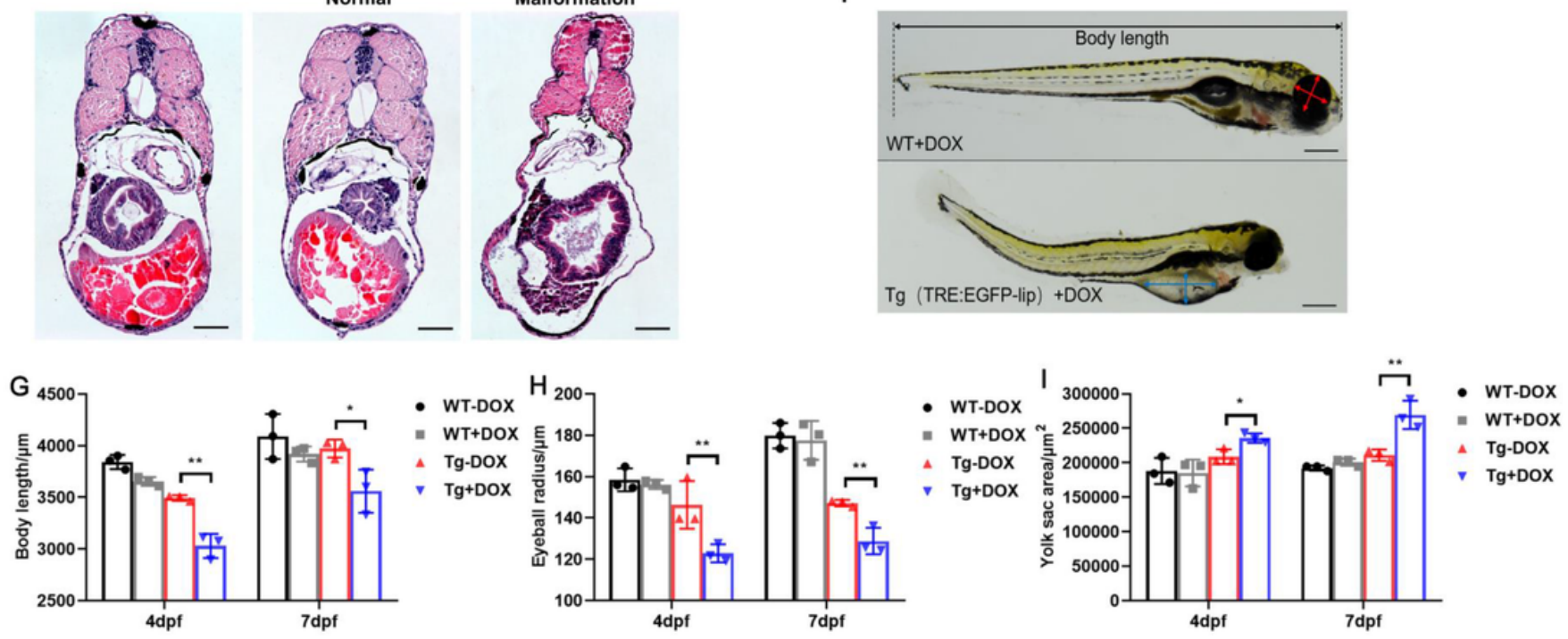

Figure 2

Overexpression of lip gene inhibits embryonic development of zebrafish. (A) The survival rates at 4-7dpf of Dox-induced wild-type and F2 embryos $(n=100)$ were calculated and compared with those of their corresponding controls. (B) The malformation rates at 4-7dpf $(n=100)$ of Dox-treated wild-type and F2 embryos were calculated and compared with those of their corresponding controls. (C) LIP overexpression causes $7 \mathrm{dpf}$ zebrafish to develop malformations. Scale $250 \mu \mathrm{m}$. (D) The ratio of various malformations of 7dpf zebrafish $(n=100)$. (E) HE staining of zebrafish larvae with LIP overexpression malformation. (F) Lateral view of zebrafish with measurements of body length, eyeball diameter and yolk sac area. (G-I): Body lengths $(\mathrm{G})$, eyeball diameters $(\mathrm{H})$ and yolk sac area $(\mathrm{I})$ of embryos injected with $\mathrm{Tg}$ (TRE:EGFP-lip) or wild-type control at $4 \mathrm{dpf}$ and $7 \mathrm{dpf}(\mathrm{n}=10)$. Data were given as means \pm standard deviation. All figures are representative of three biological replicates. ${ }^{*}, \mathrm{P}<0.01,{ }^{*}, 0.01<\mathrm{P}<0.05$ (Student's t-test). Scale bar is $250 \mu \mathrm{m}$. 


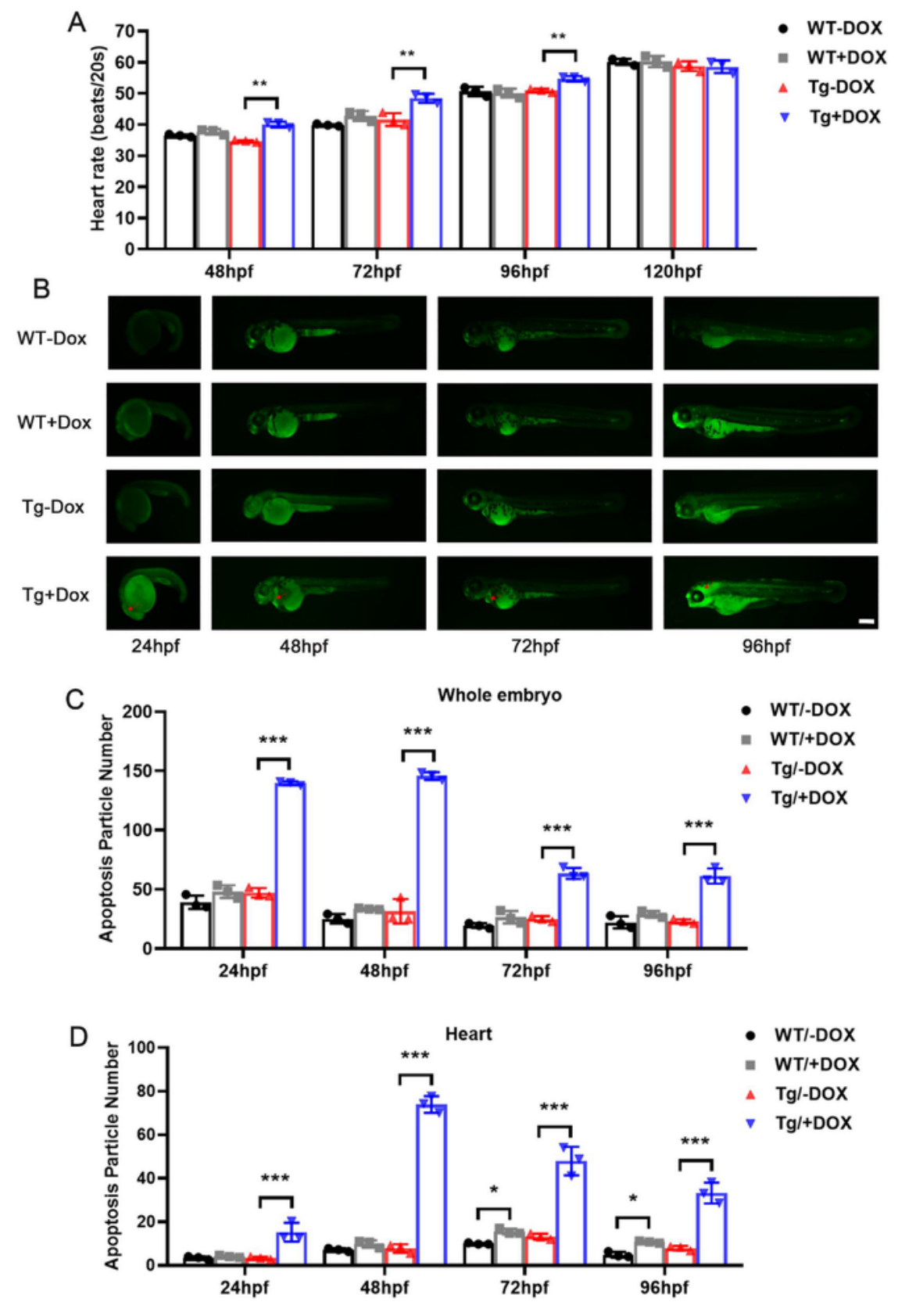

\section{Figure 3}

LIP inhibits embryonic development of transgenic zebrafish by inducing cell death. (A) Heart rate of 48$120 \mathrm{hpf}$ wild type and transgenic zebrafish every 20s $(n=10)$. (B) LIP overexpression induces effective cell death in whole embryos and heart. Wild-type control embryos and LIP overexpression embryos were stained with acridine orange (AO) at $24 \mathrm{hpf}, 48 \mathrm{hpf}, 72 \mathrm{hpf}$ and $96 \mathrm{hpf}$. Death cells are visible as bright green spots, and less bright homogenous green staining, an unspecifc background staining. Wild-type 
zebrafish exhibited few or no death cells in whole organism. In contrast, signifcantly increased staining was observed throughout the brain and eye in LIP overexpression embryos (red arrows). Lateral view, anterior, left. (C) Quantifcation of death particle number in whole embryo shows increase in LIP overexpression embryos ( $\mathrm{n}=10)$ at $24 \mathrm{hpf}, 48 \mathrm{hpf}, 72 \mathrm{hpf}$ and $96 \mathrm{hpf}$. (D) Quantifcation of death particle number in heart shows increase in LIP overexpression embryos $(n=10)$ at $24 \mathrm{hpf}, 48 \mathrm{hpf}, 72 \mathrm{hpf}$ and $96 \mathrm{hpf}$. Data were given as means \pm standard deviation. All figures are representative of three biological replicates. ${ }^{\star \star \star}, \mathrm{P}<0.001, * \star, \mathrm{P}<0.01, *, 0.01<\mathrm{P}<0.05$ (Student's t-test).
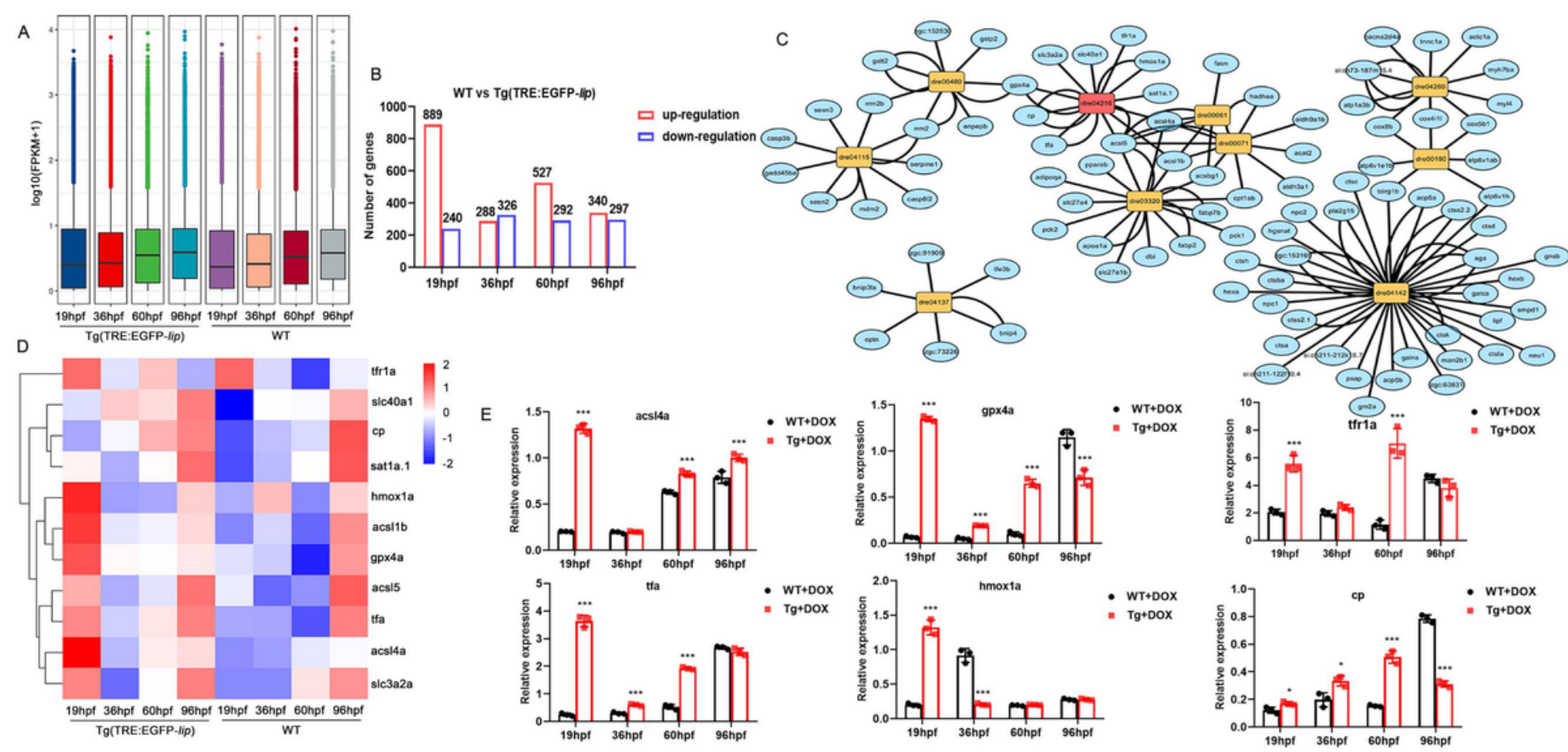

\section{Figure 4}

RNA-seq transcriptome analysis identifies a set of LIP-dependent targets in transgenic zebrafish. (A) Correlation coefficient of zebrafish at different stages of embryonic development. (B) The DEGs was upregulated and down-regulated in different periods. (C) KEGG pathway analysis of main DEGs in LIP overexpression groups, the red in the circle represent dominant influence DEG sets. (D) Heat map of RNAseq showing genes related to ferroptosis pathway. (E) Q-PCR validation of differential expression of 6 genes. In $\mathrm{E}$, data are presented as mean $\pm \mathrm{SEM}$, and all figures are representative of three biological replicates. ${ }^{* \star *}, \mathrm{P}<0.001, *, 0.01<\mathrm{P}<0.05$ (Student's t-test). 


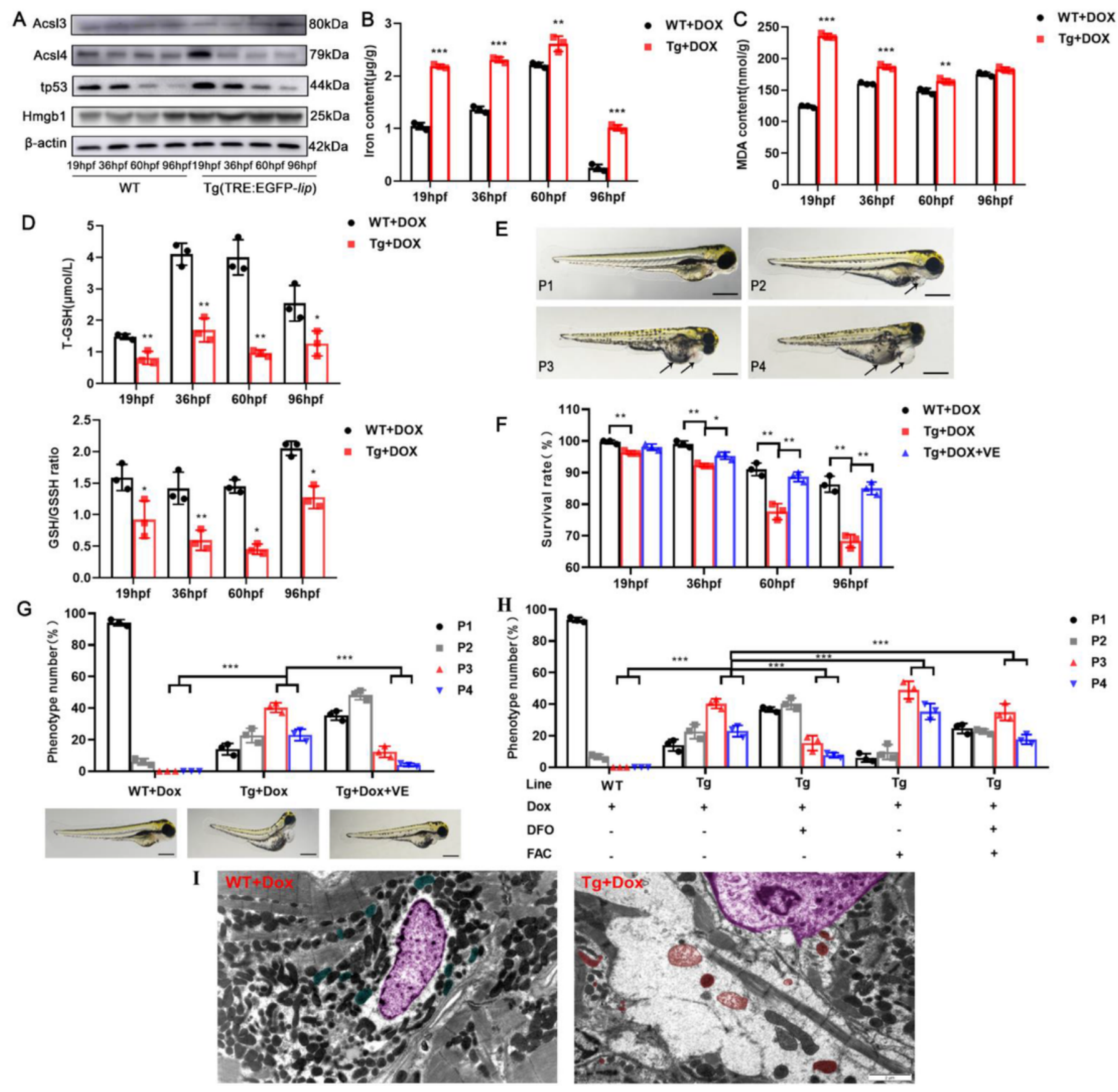

Figure 5

LIP overexpression in zebrafish larvae causes edema by triggering ferroptosis.(A) Ferroptosis expression in four stages of embryonic development from zebrafish was confirmed on Western Blot. (B-D) Zebrafish whole embryo iron content (B), MDA content (C), T-GSH content and GSH/GSSG ratio (D) detection at $19 \mathrm{hpf}, 36 \mathrm{hpf}, 60 \mathrm{hpf}$ and $96 \mathrm{hpf}$. E: Representative phenotype of the larvae categorized into 4 groups: P1 =no edema, P2 = mild edema, P3 = severe edema, P4 = very severe edema. (G) 96hpf zebrafish larvae phenotypic classification and representative images. (F) Effects of vitamin $E$ on survival rate of overexpressed LIP zebrafish. $(\mathrm{H})$ Phenotypic classification of $96 \mathrm{hpf}$ larvae treated by combination of FAC and DFO. (I) Transmission electron microscopy performed on heart sections from zebrafish myocardium demonstrating abnormal edema and mitochondria ultrastructure in LIP overexpression hearts. Pink 
means cell nucleus, blue means normal mt, red means mt after ferroptosis. Scale bar represents $2 \mu \mathrm{m}$. Data were given as means \pm standard deviation. All figures are representative of three biological replicates. ${ }^{\star \star *}, \mathrm{P}<0.001,{ }^{*}, \mathrm{P}<0.01, *, 0.01<\mathrm{P}<0.05$ (Student's t-test).

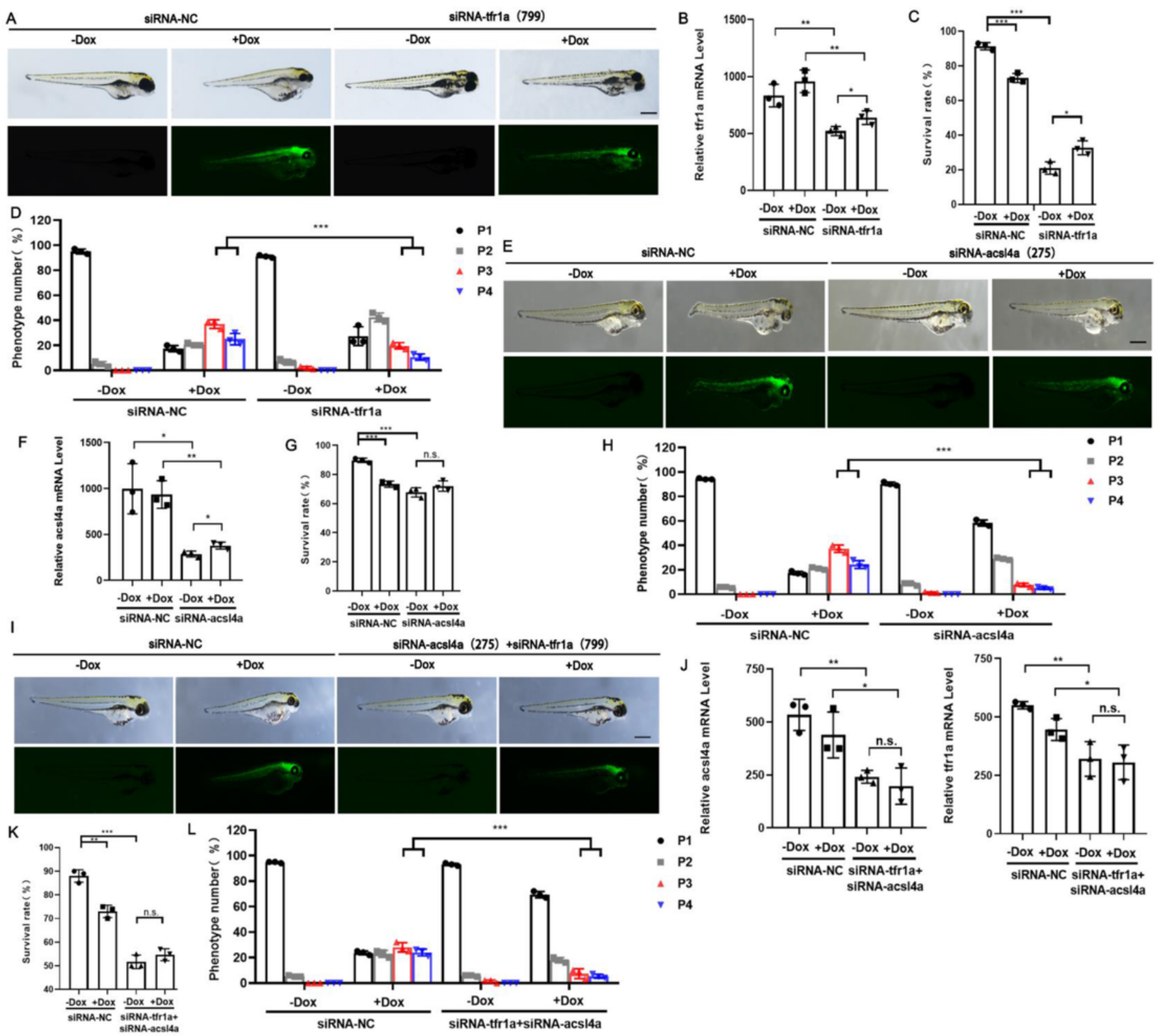

Figure 6

LIP overexpression induces ferroptosis through increase tfr1a and acs/4a. (A-D): SiRNA interference of zebrafish tfr1a. Representative phenotype (A), efficiency (B), survival rate (C) and phenotypic classification (D) of siRNA silenced zebrafish tfr1a gene expression. (E-H) SiRNA interference of zebrafish acsl4a. Representative phenotype (E), efficiency $(F)$, survival rate $(G)$ and phenotypic classification $(H)$ of siRNA silenced zebrafish acs/4a gene expression. (I-L) SiRNA interference of zebrafish tfr1a and acs/4a. Representative phenotype $(\mathrm{I})$, efficiency $(\mathrm{J})$, survival rate $(\mathrm{K})$ and phenotypic classification (L) of siRNA silenced zebrafish tfr1a and acs/4a gene expression. Data were given as means \pm standard deviation. All 
figures are representative of three biological replicates. ${ }^{\star \star *}, \mathrm{P}<0.001,{ }^{*}, \mathrm{P}<0.01, *, 0.01<\mathrm{P}<0.05$ (Student's t-test).

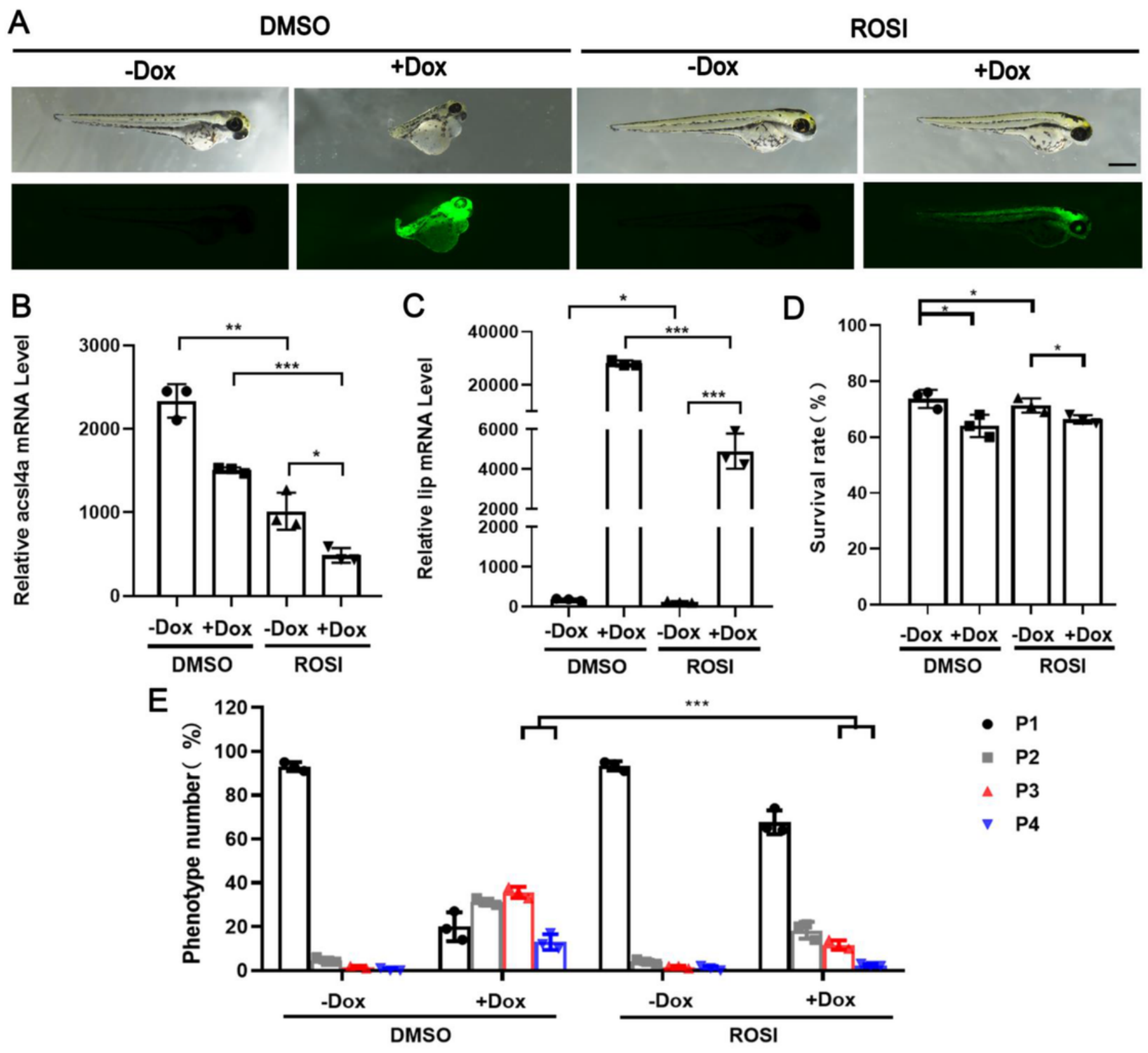

Figure 7

ROSI targeted acs/4 inhibits ferroptosis. (A) Representative phenotypes of zebrafish after ROSI treatment. (B) Inhibition of ROSI on the transcription of zebrafish acs/4a gene. (C)Inhibition of ROSI on the transcription of zebrafish lip gene. (D) Survival rate of zebrafish after ROSI treatment. E: Phenotypic classification of zebrafish after ROSI treatment. Data were given as means \pm standard deviation. All figures are representative of three biological replicates. ***, $P<0.001, \star \star, P<0.01, *, 0.01<P<0.05$ (Student's t-test). 


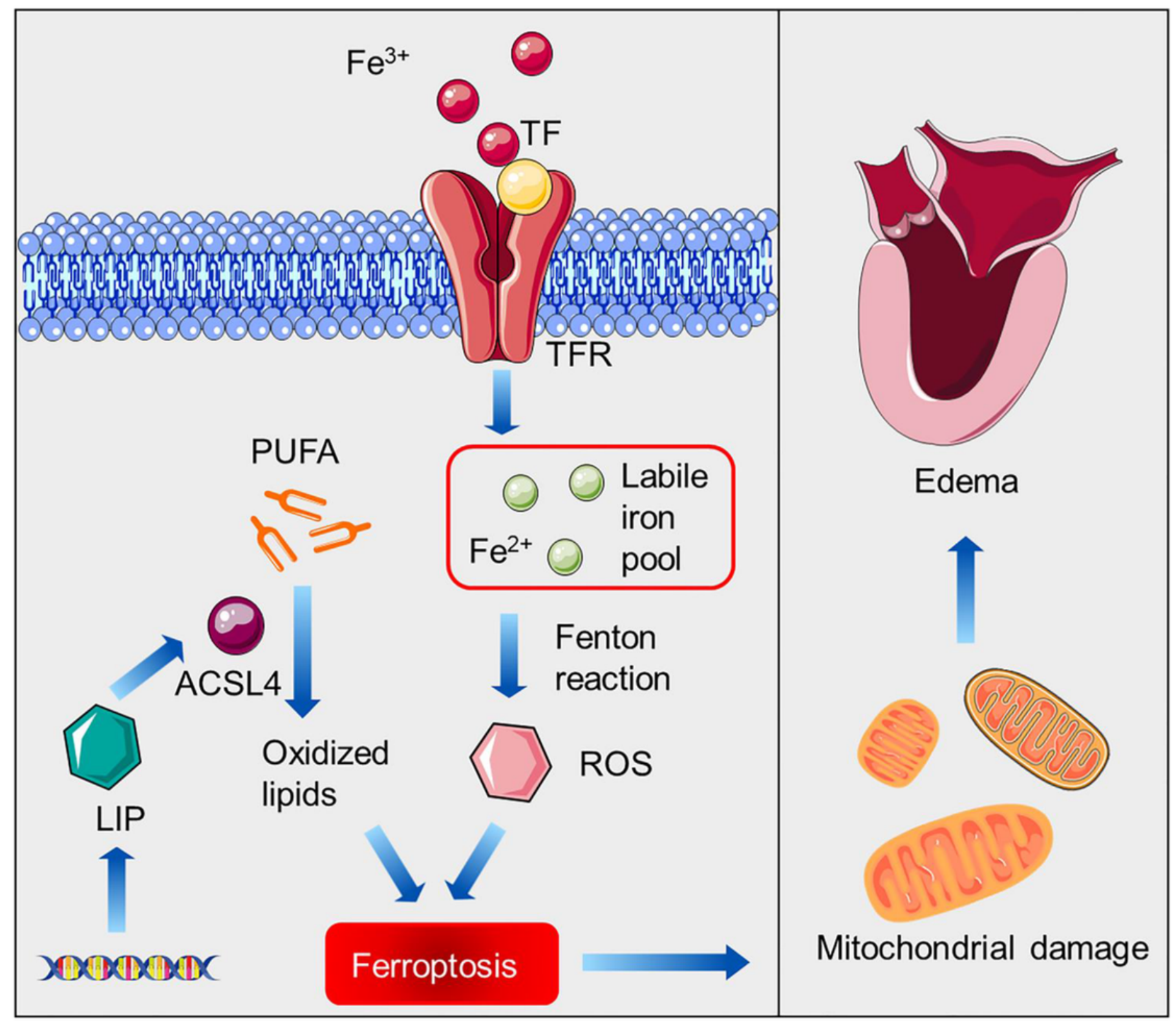

Figure 8

Network visualization of key signalling and lip gene regulatory modules that are implicated in Ferroptosis. The gene regulatory network comprises the following circuits (in chronological order of execution): 1. Iron overload, 2. Lipid peroxidation 3. Ferroptosis and 4. Mitochondrial damage and pericardial edema. Full protein names of the nodes in this network are as follows: TF: transferrin, TFR: transferrin receptor, ACSL4: acyl-CoA synthetase long chain family member 4 .

\section{Supplementary Files}


This is a list of supplementary files associated with this preprint. Click to download.

- Additionalfile1Figures1.pdf

- Additionalfile2Figures2.pdf

- Additionalfile3Figures3.pdf

- Additionalfile4Figures4.pdf

- Additionalfile5Tables1.pdf

- Additionalfile6Tables2.pdf

- Additionalfile7Tables3.pdf

- Additionalfile8Tables4.pdf 